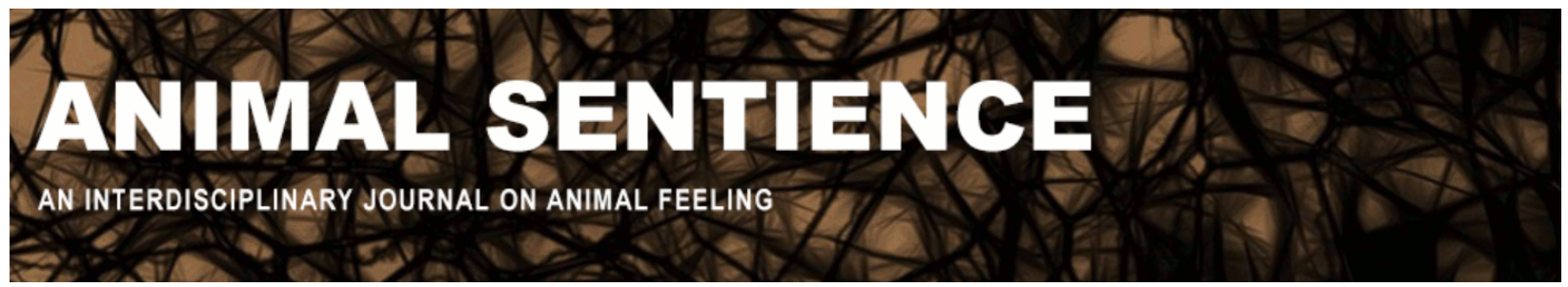

Powell, Russell and Mikhalevich, Irina (2020) Affective sentience and moral protection. Animal Sentience 29(35)

DOI: $10.51291 / 2377-7478.1668$

Date of submission: 2021-01-09

Date of acceptance: 2021-01-09

(c)

This article has appeared in the journal Animal

Sentience, a peer-reviewed journal on animal

cognition and feeling. It has been made open access,

free for all, by WellBeing International and deposited

in the WBI Studies Repository. For more information,

please contact

wbisr-info@wellbeingintl.org.

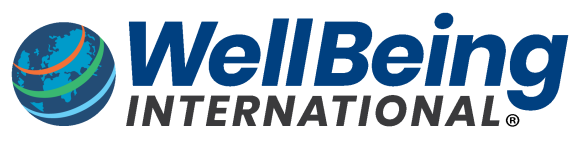

SOLUTIONS FOR PEOPLE, ANIMALS AND ENVIRONMENT 


\title{
Affective sentience and moral protection
}

Response to Commentary on Mikhalevich \& Powell on Invertebrate Minds

\author{
Russell Powell \\ Department of Philosophy, Boston University
}

Irina Mikhalevich

Department of Philosophy, Rochester Institute of Technology

\begin{abstract}
We have structured our response according to five questions arising from the commentaries: (i) What is sentience? (ii) Is sentience a necessary or sufficient condition for moral standing? (iii) What methods should guide comparative cognitive research in general, and specifically in studying invertebrates? (iv) How should we balance scientific uncertainty and moral risk? (v) What practical strategies can help reduce biases and morally dismissive attitudes toward invertebrates?
\end{abstract}

\begin{abstract}
Russell Powell, Associate Professor of Philosophy, Boston University, specializes in philosophical problems in evolutionary biology and bioethics. Website
\end{abstract}

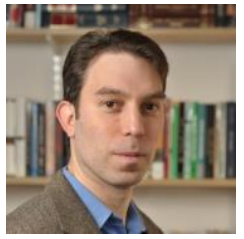

Irina Mikhalevich, Assistant Professor of Philosophy, Rochester Institute of Technology, specializes in conceptual and methodological problems in comparative cognitive science and their implications for the treatment of nonhuman animals. Website

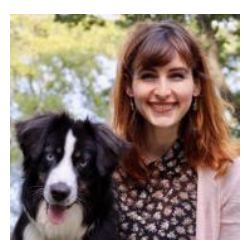

We are grateful for the thoughtful attention that each of the thirty-three commentators (so far) has given to our target article during these difficult times. Although considerable disagreement remains about the structure of sentience, how it should be studied, its moral significance, and its distribution in the living world, it is heartening to see so many thinkers from such disparate disciplines and intellectual traditions take the possibility of arthropod minds and moral significance seriously. We take comfort in knowing that despite our lingering disagreements, we all share the same goal: to ensure that our interactions with animals, whether in the laboratory, in agriculture, in the marketplace, or in our daily lives, are guided by the best available empirical evidence and that our actions live up to the high standards that morality requires. We hope that engaging with these unresolved questions, albeit briefly and incompletely, will move the discussion forward and allow us to resolve some confusion about our own view along the way. 


\section{The Nature of Sentience}

1.1 The Role of Affect. The standard practice for this journal is to equate "sentience" with feeling (anything); a sentient state is a felt state. Philosophers call such states "phenomenal consciousness" ("PC"). Many of the commentators on our article adhere to this definition of sentience (see, e.g., Levy; Marino; Mallatt \& Feinberg; Cartmill; Woodruff; Dietrich \& Fox Hall; Veit \& Huebner). The view we advanced in our target article is that for moral purposes sentience must involve positively or negatively "valenced" feelings (also called "affects"), not simply neutral sensations. In our view, it is affect that distinguishes beings who merely perceive or represent internal or external states from sentient beings who can feel good or bad about those states-and who can therefore be helped or harmed by the actions of moral agents. It is affect that makes sentience matter.

Mallatt \& Feinberg urge us to adopt the view that sentience is the same thing as PC on the grounds that PC already includes affect. But their own partition between affective consciousness and sensory consciousness draws precisely the sort of distinction that we think is important for moral purposes. If it is possible to have sensory consciousness without affective consciousness, as their distinction implies, then sentience per se is not sufficient for moral standing. And if the presence or absence of sentience in an organism is to have practical consequences for animal ethics and policy, as it currently does, then the focus should be explicitly on valenced feeling. It is for this reason that when we use the term "sentience" in this Response, we will be referring specifically to affective sentience.

1.2 Sentience as a Value-Laden Concept. In attempts to describe the causal structure of the world, scientific categories should lead and moral conclusions (if any) should follow. However, in some cases scientific concepts are so intertwined with normative thought, institutions, and practices that specifying them needs to take moral considerations into account. (This is true, for example, of the concepts of 'disease' and 'death' in human medicine). The prevailing concept of sentience in ethics is closely linked to notions of welfare and is used to guide bioethics and policy. Its ability to do moral work hence needs to be taken seriously. That this journal takes the ethical implications of sentience to be central to its intellectual mission supports our view that sentience should be conceived in a way that is suited to its normative role-and this, in turn, requires that sentience be affective.

This is not to commit the naturalistic fallacy in reverse, inferring how things really are from how they ought to be. Rather, it is to specify which particular biological properties are at issue for ethics; then empirical research can go on to identify real world instances of those properties and policy can apply the findings. Degrazia, a leading theorist of moral status and animal ethicist, agrees that it is affective sentience that matters morally, and has put forward a similar argument himself-The emphasis on valenced feelings shifts attention away from elusive notions like PC toward more empirically tractable concepts in welfare research, such as emotion, affect, pain, pleasure, desire, preference, and so on.

1.3 Does Sentience Admit of Degrees? One question that arose in the commentary is whether sentience is an all-or-nothing phenomenon or admits of degrees. In the target article we suggested that sentience, like other complex biological traits, is likely to be continuously distributed in nature. We were not clear, however, about how this continuous distribution relates to the question of gradation. The issue is this: How can sentience be an all-or-nothing 
property and at the same time be continuously distributed in the natural living world? Lee takes a significant step toward answering this question. The crux of the issue, as Bayne et al. (2016) put it, is that "One person can be conscious of more objects and properties than another person, but to be conscious of more is not to be more conscious." Lee's framework allows us to say that the experiential content of sentience can vary in degrees and types, even if sentience itself, like pregnancy, is a binary phenomenon. This accords with Marino's point that "more complex vertebrate brain structures may simply change the content or increase the range of features in the world we are aware of, but they are not necessary for basic subjective experience."

Even if gradations in content are unlikely to generate a quantifiable scale of consciousness, however, the "richness" of sentience will tend to correspond to greater constraints on ethical treatment. It is easier to satisfy the interests of an organism that is only sensitive to and cares about one or a few things than it is to take into account the preferences of one that is sensitive to and cares about many different kinds of things. The content of sentience may thus have important consequences for ethical treatment, whether or not it results in higher levels of moral status or morally weightier interests (more on this below).

\section{The Basis of Moral Standing}

2.1 How is Cognitive Capacity Related to Moral Standing? Let us now consider how evidence of cognitive capacities might bear on moral standing. One possibility is that the presence of certain cognitive capacities implicates sentience and hence moral standing. We will call this the "implication" view. This is the prevailing view among the commentators, many of whom appear assume that sentience is necessary and sufficient for moral standing and that cognition research is currently the best tool we have to probe for the presence of sentience.

An alternative view is that the relation between cognitive capacities and moral standing is a direct one-namely, that certain cognitive states, if present, are sufficient for moral standing whether or not they are accompanied by feelings. A motivation for and a purported advantage of this "direct relation" view is that it avoids the perceived methodological difficulties of inferring internal felt states from observable behavior (see Cartmill; Dietrich \& Fox Hall), difficulties that are even more pronounced in the invertebrate context. Levy, for example, proposes that the capacity to have beliefs and desires is sufficient for moral standing: an individual can have moral standing without having neutral feelings, valenced feelings, or any feelings at all. But depending on how one construes "beliefs" and "desires," these mental state categories may run into inferential difficulties of their own. Levy's point, presumably, is that these difficulties are more tractable empirically than, say, the problem of 'qualia' or 'other minds. Delon et al., too, argue that "agency" - capaciously defined as a motivational and goal-representing system that results in flexible behavior-is enough for moral standing. On their view, however, sentience is morally relevant insofar as it acts as a value-enhancer (see also Howard \& Symonds).

The problem with these "direct relation" views, however, is that in the absence of feelings, goal-directedness and the capacity for beliefs and desires-and, as we have suggested, even affectively neutral sentience-do not matter morally. Robots, plants, slime molds, and bacteria can "represent" goals in some non-conceptual sense, and they can behave flexibly to some degree-but they lack the feelings that make the frustration of their 
goals frustrating or the achievement of their goals rewarding. By the same token, artificial intelligences may be capable of complex computations and even of forming beliefs, and yet incapable of wanting or caring about anything (McShea 2016). Our central point is that in the absence of affective experience, beliefs and desires do not give rise to subjective harms, and thus moral status cannot be derived from these mental categories alone. Of course, belief-desire capacities and agentic behavior may indicate the presence of sentience, but this is a far cry from the notion that moral standing arises from these cognitive capacities in and of themselves.

If it could somehow be shown that all beliefs and desires are affectively valenced, then investigations of cognition would yield results about affective sentience, and hence moral standing. However, there is a more direct route to establishing moral standing, and that is the empirical investigation of affective states. Even if the functional markers of valence are harder to discern for the "alien" brains and behavior of invertebrates (Baracchi \& Baciadonna), affective states, as Broom points out, are describable functionally and hence within the realm of scientific analysis. There is thus no methodological reason to exclude affect from the set of capacities that are necessary or sufficient for moral standing, and there are sound reasons (as we saw earlier) for including it.

Even if one accepts (as we do) the notion that cognitive capacities are relevant to moral standing only insofar as they implicate sentience, many cognitive capacities will still have no bearing on sentience and thus on moral standing. Several authors (Vonk; Howard \& Symonds) took us to be arguing that once certain sophisticated cognitive abilities have been demonstrated in a lineage - such as spatial cognition, numerosity, logical proficiency, the use of abstract concepts, problem solving, memory, etc.-this is sufficient to establish moral standing. This is not our view. We think that cognition is only relevant to moral standing insofar as it implicates affective sentience, and that if sophisticated cognition generates a higher moral status, it only does so once certain thresholds of "psychological personhood" are reached (see 2.3 below). In our target article, we explicitly state that even impressive cognitive feats need not bear on the presence of sentience, and hence on moral standing, although some cognitive abilities (such as open-ended associative learning, if it has the implications for affective sentience hypothesized by Ginsburg \& Jablokna 2007) may do so. We thus agree with Vallortigara that cognitive complexity has no straightforward implication for ethics. We also state clearly in the target article that although we take welfare to be a psychological property, psychological capacities in and of themselves are not sufficient for welfare-the capacity to have pleasurable and aversive experiences is also needed. This will prove important in deciding how far down the slippery slope toward panpsychism we need to go.

2.2 The Slippery Slope to Panpsychism. Some commentators, such as Figdor, suggest that "psychological traits" may be present in brainless animals and even in organisms with no nervous system at all, such as plants and unicellular organisms, and that this may be sufficient for moral standing. We are not convinced that there is evidence for psychological capacities (or for mental states such as beliefs and desires) in brainless or non-neural organisms even if they do exhibit some minimal memory, learning, and problem solving (Mallatt \& Feinberg, Marino, and Cartmill are in accord). But even if it were agreed that chemotaxis in bacteria (Adler 1966), maze-navigation and puzzle-solving by protists (Reid et al. 2012), or habituation in mimosa plants (Abramson 2016) show that these organisms 
are genuinely cognitive beings, this would not show that they have valenced feelings of the sort that give rise to welfares in the morally relevant sense. As we stressed above, our view is that affective sentience is a necessary condition for having a welfare, and there is no evidence at present for feelings of any kind in green plants, fungi, or unicellular organisms.

Dietrich \& Fox Hall go further to suggest that all living things may not only have cognitive capacities but they may also all be sentient, where "sentient" for them means either neutral or valenced feeling. Even more radically, they maintain that given our limited understanding of the structure of conscious experience, even panpsychism is a perfectly respectable philosophical position. Panpsychism is the view that mentality is built into the very furniture of the universe, with experiential qualities arising from basic material objects like atoms. We do not need to invoke the many objections to panpsychism (such as the unresolved problem of how the purported 'minds' of sub-atomic particles combine to form the more familiar ontologies of macroscopic animal minds) because on our view neutral feeling alone would not be sufficient for moral standing. Reserving moral standing to beings capable of affective sentience avoids slipping into the ethical problems of biopsychism and panpsychism.

Broom argues that the concept of welfare applies to a broader range of organisms than merely those that are sentient or have cognitive capacities (Key \& Brown make similar suggestions). Broom suggests that non-psychological welfare may give rise to a lower moral status than does sentience. In our target article, we distinguish between biological welfare and psychological welfare and remain skeptical that a purely biological welfare could give rise to moral standing in any meaningful sense. The moral implications of sentience are of a radically different order from those (if any) that stem from just being alive. For a recent analysis of the problems with biocentric accounts of moral standing, see Basl (2019).

2.3 Moral Status and Triage. Another way of avoiding a moral slippery slope is to reject the idea that either sentience or cognition is sufficient for moral standing, and instead reserve membership in the moral community for beings who have rationality or some other higherorder cognitive property. Key \& Brown appear to take this tack. Unfortunately, they erroneously link moral standing with legal personhood, conflating distinct concepts that have been developed in moral, political, and legal philosophy. Legal personhood, whether natural or juridical, is the basis of legally prescribed rights or privileges. Moral standing, whatever its basis, can provide moral reasons for protective action (or inaction) whether or not these protections are codified in or otherwise recognized by law.

Psychological personhood, in contrast, refers to higher-order properties such as the capacity for rationality, mutual accountability, interests that extend into the future, and so on. As Degrazia notes, psychological personhood may result in a higher moral status, or in interests that are accorded greater priority in triage scenarios such as biomedical research (see also Buchanan 2011; McMahan 2009; Agar 2013). Few moral theorists today, however, believe that personhood is necessary for moral standing. This is true not only for Utilitarians but also the vast majority of contemporary ethicists working in the Kantian tradition (Powell, Mikhalevich, \& Buchanan in press), nearly all of whom agree that moral standing entails, at the very least, that sentient beings should not be made to suffer needlessly.

Clarke argues that our view of moral status results in an apparent contradiction: that on the one hand we say that invertebrates have lower moral status than vertebrates, but on the other hand, we say that invertebrates should not replace vertebrates in scientific 
research. This misinterprets us on both counts. We do not conclude that all invertebrates with moral standing have a lower moral status than any vertebrate (or any vertebrate with moral standing). Indeed, we do not know how moral status could be compared at that grain of resolution. But neither do we reach the conclusion that invertebrates with moral standing should never replace their vertebrate counterparts in research (Soryl misreads us similarly on this point). We argue against a blanket replacement policy that rests on evolutionary anachronisms, cognitive biases, and inconsistent readings of the empirical evidence. Neither the question of relative moral status nor that of replacement is amenable to categorical answers; they must be addressed on a case-by-case basis.

It is important to note, however, that the fact that one group has a lower moral status than another does not mean that in situations of conflict their interests are always trumped by the interests of beings with higher moral status. For example, some agricultural animals may have a lower moral status or less weighty interests than full human persons, but that does not mean that humans are morally permitted to farm those animals without regard for their wellbeing. It is also possible that the needs of many beings with lower moral status could, in the aggregate, trump those of a smaller number of beings with higher moral status (Crisp 2020). We will return to this "aggregation problem" later on when we discuss the consequences of recognizing the moral force of invertebrate welfare (§4.3).

2.4 Invertebrates Challenge the Traditional Welfare Ethics Paradigm. Other commentators (Monsó \& Osuna-Mascaró and Forber \& Jones) advocate a welfare-based theory of moral standing but highlight some of the complications that arise in extending the traditional individual welfarist paradigm to very different organisms such as insects, where the ontology of the individual stretches beyond familiar parameters. Monsó \& OsunaMascaró point out that some insects, like the caste members of eusocial colonies, may be less-than-individuals, whereas other insects, such as those that undergo radical metamorphoses, may be viewed as different individuals at different times in their life cycle.

Metamorphosis creates problems that are analogous to those surrounding the continuity of personal identity, for moral purposes, from fetus to adult or following traumatic brain injury or illness. We will not discuss these thorny problems here, apart to note that that similar difficulties already confound human bioethics.

Biological individuality at the colony level raises the interesting prospect that welfares obtain at higher levels of evolutionary organization. Eusocial insect colonies may be proper evolutionary individuals or "superorganisms," with castes that have been cognitively "simplified" in virtue of their specialized roles in a way that is analogous to cell line specialization in animal bodies. This does not, however, imply that the colony has a welfare that is not reducible to that of its insect components. The superorganism may have a "biological" welfare of its own in the same way that other living things do, and it may have greater information processing powers, better problem solving, and more flexible behavior than is found among its individual parts. Perhaps we could even attribute beliefs and desires to the hive. This is a far cry, however, from the notion that superorganisms are psychological individuals in any meaningful sense, let alone that they are sentient in the affective sense that matters for moral purposes.

Determining whether eusocial colonies are sentient requires a much better understanding of its mereological structure (i.e., part-whole relationships) than we now have. Tononi and Koch (2015) reject the possibility of sentience in aggregates of sentient 
individuals, whereas Schwitzgebel (2015) argues that even loose aggregations of individually sentient individuals, such as nations, have some of the hallmarks of sentience. Theorists of consciousness have often alluded to the eusocial colonies and the so-called "hive mind," sometimes to illustrate the fuzzy ontological boundaries of sentient systems and sometimes to argue that centralized information integration is a precondition for felt experience. Some have even used the eusocial colony as a test case for scientific theories of consciousness, (e.g., Friedman Søvik 2019). Much work remains to be done on the question of hive sentience.

2.5 Is Our View of Moral Considerability Anthropocentric? Some commentators worry that taking sentience, or even just psychological properties, as the basis of moral standing is unduly anthropocentric (see Figdor; Fitzpatrick; Vonk). It is true that our view of moral standing is not maximally inclusive: it does not include life forms that lack psychological traits altogether. But our approach is fundamentally anti-anthropocentric in that it views sentience as a basal property of several animal lineages that evolved centralized nervous systems convergently in the early phases of animal evolution (a view shared by Mallatt $\boldsymbol{\&}$ Feinberg; Marino; see also Powell 2020). There are good reasons for linking sentience to moral standing beyond the mere fact human beings (whose intrinsic value is taken as a given) are sentient.

Fitzpatrick objects that we (as authors or humans) simply grant moral status to some lineages while denying it to others, as if these were declarations. But in prevailing views of moral standing, moral agents do not "grant" moral standing to moral patients or to other moral agents; rather, they recognize or acknowledge moral standing in the world. This must be done on the basis of rationally defensible (non-arbitrary) reasons that are applied consistently across similar cases, resulting in what Lecky (1921) called the "expanding circle" of moral concern.

That we begin this analysis with the human case-identifying sentience as the source of our own moral standing and then asking where else sentience may be found-does not bias our inquiry as long as our subsequent analysis is consistent, reasonable, and defensible. There is a vast philosophical literature exploring why psychological welfare matters morally. The welfarist view of moral standing is well-developed and widely implemented in law and policy. Rival theorists must propose an alternative that constrains behavior in the real world at least as meaningfully. We have already seen that merely the property of being alive will not suffice, for even if being alive matters in some vaguely moral sense, it is not evident why it would matter in the self-evident way that positive and negative feelings like pain and pleasure matter.

\section{Evidence, Inference, and Methodology}

3.1 Another Look at the Evidence. We are grateful to many of our commentators for strengthening our empirical analysis with interesting and helpful examples (e.g., Cammaerts; Baracci \& Baciadonna; Elwood). Although most found our analysis of the literature on arthropod sentience persuasive, some did take issue with our interpretation of the empirical evidence. For example, Key \& Brown cite the fact that some human behaviors can be performed unconsciously as grounds for skepticism about the behavioral evidence of sentience in invertebrates (Cartmill makes a similar point about sleepwalking). In our target 
article, we highlight the problem of unconscious behavior and learning and agree that it poses a challenge to inferring sentience in non-human animals. However, we do not claim that the behavioral evidence we canvass is conclusive proof of sentience: Rather, we argue for consistency in how behavioral evidence of sentience is interpreted across Metazoa. If certain behaviors in vertebrates count as evidence of sentience sufficient for moral standing, then evidence of similar behaviors in invertebrates should likewise count as sufficient evidence. We agree with Baracci \& Baciadonna that "if cephalopod mollusks have been considered 'honorary vertebrates' for their cognitive abilities, it would seem logical, if not imperative, to attribute the same status to bumblebees and honeybees, based on the evidence available."

Key \& Brown and Forber \& Jones, are mistaken that our argument for consistency entails the reciprocal possibility that vertebrates lack sentience through what might be thought of as our consistency argument in reverse. The logic of this "reverse consistency argument" is something like the following: If the data are insufficient to establish sentience conclusively in invertebrates, then consistency entails that the same should be true for vertebrates; hence we ought to be agnostic about vertebrate sentience. This reasoning is invalid for two reasons. First, agnosticism does not follow from the lack of scientific conclusiveness. Second, there is abundant evidence for sentience in vertebrates that goes well beyond the behavioral experiments that have been replicated in invertebrates.

Let us elaborate on the first point. Forber \& Jones write that we "make a clear declaration about the circumstances under which attributing sentience is justified." They then quote the following passage from our target article: "Until we have a fully worked out and empirically corroborated theory of the relationships among neural tissue, cognitive processes, and phenomenology, we are not in a position to rule out or even deem unlikely the possibility that tiny brains can give rise to sentient lives with interests that merit moral protection." Like Key \& Brown, Forber \& Jones are misreading our argument. The claim we make in the quoted passage is about the conditions under which sentience can be ruled out for small-brained invertebrates; we do not claim, here or anywhere, that a fully worked out theory of consciousness or brain-mind relations is necessary for the scientific or moral attribution of sentience. We argue at length in the target article that ethical risk management does not require such strong evidence. Nor do we suggest that in the absence of a full-blown theory, all invertebrates should be presumed to have sentience. We dedicated a substantial portion of our paper to reviewing the empirical evidence for sentience in particular invertebrate groups.

3.2 The Evidential Role of Homology. There remain key unanswered questions regarding the phylogeny of animal nervous systems. If we cannot be reasonably certain whether the last bilaterian common ancestor had distributed nerve nets, simple ganglia, or a hierarchically structured brain, we cannot rely on homology to make inferences about sentience across metazoans (Powell 2020). Key \& Brown maintain that our argument for treating the evidence consistently does not work for animals whose brains lack structural homologies with human brains. They write: "The two cases are not symmetrical...because we have good reason, on the basis of structural homologies, to conclude about many vertebrate species that they are conscious." In the target article, we discussed various problems with privileging evidence from homology in making inferences about sentience, such as the possibility of convergent neurocognitive evolution, the likelihood that some of 
the neural correlates of consciousness in humans were co-opted from more ancient structures, and the fact that homologies are not themselves "function-free." We will use this opportunity to elaborate on the last point, because it seems to have been misconstrued.

In the target article we note that homology is often treated as evidentially privileged over homoplasy because homology hypotheses, in contrast to convergence hypotheses, supposedly pick out ancestral similarities without making any functional claims. Homology judgments, however, are not themselves function-free; hence they lack the supposed evidential advantages over convergence hypotheses (see Woodruff). Key \& Brown mistakenly interpret our claim that homologies are not function-free to mean that the homology of two similar neural structures is determined in part by the functional behavior they produce, which they note is "wildly out of step with fundamental principles of comparative neurobiology." That is not our argument. Our argument is that in order to establish that two neural structures are homologous, we must carry out an evolutionary neurobiological analysis, i.e., a functional one: this removes the main perceived advantage of homology in drawing inferences about sentience.

We can elaborate a bit further without plunging too deeply into this complicated issue. Many theorists argue that biological traits are distinguished in virtue of their functions. The "function" of a trait is defined either etiologically in terms of its history of selection for certain effects (Neander 2002; Rosenberg \& Neander 2009; Neander \& Rosenberg 2012) or a-historically in terms of the causal roles it plays in some capacity of a complex system (Amundson \& Lauder 1994). Analyses of functional integration are central to the work of comparative morphologists. Some homologous traits are themselves types of adaptations: Bird forelimbs are homologous not only as vertebrate forelimbs but also as wings. In cases where there are large differences between two traits in form and function-as with the inner ear bones of mammals, the jaw hinges of fish, and the gill arches of still other fish-homology can be determined in part by transformations of functions as reflected in a series of intermediary structures recorded in the fossil record. There is ongoing debate among theoretical biologists over which type of function is most important in homology assessment, but it is well established that homology considerations are inescapably intertwined with functional analyses. Homology and convergence are both highly inferential and require functional hypotheses; hence they are on comparable epistemological ground.

A different way to counter the homology objection to invertebrate sentience is to argue that there is extensive homology between vertebrate and invertebrate nervous systems, at least at physiological and molecular levels (see Marino). The inference of emotion-like states in bees, for example, is supported by the presence of neurotransmitters like dopamine that regulate emotion in humans and other vertebrates. Molecular homology alone, however, offers limited insight into macroscopic functions. Consider the role of the Pax-6 gene (and its orthologues), which plays a key role in the development of eyes in both vertebrates and invertebrates (including arthropods and mollusks). The mere presence of Pax-6 is not strongly correlated with the presence of eyes, however, since the gene can be found in many eyeless (and brainless) animals, such as echinoderms and nematodes, where it is associated with the anterior patterning of the head. Pax- 6 appears to have arisen prior to the development of eyes and was only later co-opted for vision-related functions. The lesson is that molecular homologues may have very different functions in disparate animal groups; and in the absence of functional analyses, they reveal little about the presence of sentience. 
We agree with Brown and others who see a parallel between how the homology argument is used against invertebrate sentience and how it is used to motivate skepticism about pain perception in fish (Rose 2002, Key 2016). Similar arguments have been raised to cast doubt on complex cognition in other animals, such as birds (Pepperberg 2009). Birds lack neural structures that are homologous to the 6-layered mammalian neocortex; this motivated the search for "simpler" interpretations of early behavioral evidence of sophisticated cognition in parrots and corvids. The weight of the behavioral evidence, coupled with advances in neurobiology, eventually forced avian neurobiologists to redefine regions of the bird brain according to their function (Jarvis et al. 2005). This shows the role of functional analysis in understanding neuroanatomical traits. The same skeptical strategy is used in the fish pain debate: the absence of brain homology is used to support an asymmetrical interpretation of the behavioral evidence, which then opens the door to nonsentient interpretations.

Key \& Brown's objections here are identical to those that Key (2016) made in his own target article arguing against fish pain. As many excellent commentaries on that article pointed out, we ignore the possibility of convergence at our peril (Brown 2016; 2020; Dinets 2016). Dinets pithily explains that "automatically extrapolating the similarities among some mammals to non-mammalian taxa would lead to outright absurdities such as that crickets are deaf because they don't have human-like ears complete with eardrums; birds can't eat solid food because they lack teeth; and fish cannot move because they don't have legs." The cortex-centered homology argument has been bolstered by scala naturae-type assumptions that, as Figdor points out, still operate in some quarters of neuroscience (Jarvis et al. 2005). Many still regard basal vertebrate brains as primitive, stripped-down versions of mammalian brains. This mistakenly treats the brains of non-human vertebrates as frozen steps in a ladder of brain evolution, rather than as features of equally modern lineages that may also be subject to complexifying selection. If any theory is guilty of the anthropocentrism that many of the commentators rightly lament, it is that sentience is limited to those neural structures that give rise to sentience in humans.

3.3 Anthropomorphism and Lloyd Morgan's Canon. It is suggested by Key \& Brown that in using terminology like "pleasure" or "aversion" we are guilty of circular reasoning. They urge us to use neutral language that does not presuppose the very answers we are investigating. We are not, however, drawing conclusions about pleasure and aversion $a$ priori. If we use affective language (e.g., that bees appear to experience shaking as aversive) we do so on the basis of the behavioral evidence. Moreover, "neutral" language is rarely neutral and runs the risk of circularity. If one adopts non-sentient terms for (say) bees or fish but not rats or primates, one is likely assuming that the non-sentient conclusion is more likely to be true for the former than the latter. This risks setting up a double standard in which the same behavior comes to be labelled in different ways for different animals. This is precisely the crux of our complaint.

The search for neutral language harks back to the behaviorist view in comparative cognition that mental-state terms (pleasure, belief, desire, etc.) are not only sentimental and unscientific, but anthropomorphic (Kennedy 1992). Such views have since given way to cognitive theory, using cognitive terms (Figdor), and for good reason. Consider the anthropomorphism objection. Attributing human-like properties to animals is only wrong if we have evidence that they are exclusive to humans-and that is an empirical matter. 
Anthropomorphism, understood as the mistaken attribution of human properties to nonhuman entities, is therefore no less wrong than what deWaal (1999) calls "anthropodenial," or the mistaken failure to attribute a human property to nonhumans. It does not make sense to guard against one type of error but not the other. We agree with Sober (2005) that the solution is not an armchair preference for one kind of error, but a strategy for avoiding both in equal measure. The central error in both anthropomorphism and anthropodenial lies in assuming human beings to be the measure of all things-in other words, anthropocentrism (Chapman \& Huffman 2018; Andrews 2020).

A similar problem arises for the strategy of erring on the side of the simplest possible explanation of animal behavior. "Simplicity" concerning cognition is often explicitly defined in reference to human cognition. A cognitive state is considered to be more "complex" when it resembles the cognitive abilities characteristic of human beings, such as reason, language, abstract thinking, mathematical abilities, and so on. The preference for simpler explanations then takes the form of the injunction against anthropomorphism.

This 'simplicity heuristic' is a long-standing tradition in comparative cognition. It can be traced back to an interpretation of a passage from a founding text in the field that has since become known as "Lloyd Morgan's Canon" (Morgan 1893). Zentall reads us as arguing that the simplicity heuristic should not be used because it will tend to result in the underattribution of sentience. We did not reject the simplicity heuristic in our target article, nor suggest rejecting it because it leads to the mistreatment of animals. If there are reasons for rejecting the simplicity heuristic, they must be grounded in the fact that it leads science astray (see Mikhalevich 2014/2015/2017). Our point was that ethical analysis must in some way acknowledge the fact that the widely-adopted simplicity heuristic leads to the moral risk of systematic false negatives.

3.4 The Adaptive Value of Sentience. In our target article we draw on evolutionary evidence to bolster the case for invertebrate sentience. If pain perception is adaptive, for example, then there is reason to expect pain-associated behavior to accompany the feeling of pain following an injury and vice versa. There can sometimes also be adaptive reasons for the absence of pain-associated behaviors (e.g., withdrawal, grooming) and the persistence of normal behaviors (e.g., feeding, mating) after injury. Elwood and Gibbons \& Sarlak suggest that some insects may have pain perception systems that are suppressed during mating, courtship, or feeding in order to enhance fitness. Gibbons \& Sarlak further note that hunger suppresses pain responses even in mammalian vertebrates, perhaps because eating supplies the energy that an injured organism needs in order to heal. The behaviors that accompany pain perception may also depend on social context. In some highly social species it may be adaptive to signal pain when one is in the presence of nest-mates (Gibbons \& Sarlak) or group members, but not among strangers, competitors, or predators; in other social ecologies, pain behavior could lead to social ostracism or decreased mating opportunities. This all points to the importance of interpreting behavioral responses to injury in an adaptive ecological context.

Cartmill raises an objection to adaptive reasoning about sentience, citing a paper in which he poses the following 'Darwinian dilemma': "If expressive behaviors have no adaptive value, then natural selection cannot account for them. But if expressive behavior is adaptive, then it cannot furnish evidence for animal awareness" (Cartmill 2000). If this were true, it is not clear to us why we could not say the same for human pain, for loving our children, for 
our fondness of sweets, or for seeing the color red. These experiences are adaptive in primates and yet we know them to be felt states. The fact that a trait is adaptive has no bearing on whether it is felt. Once we see these explanations as operating at different levels (i.e., the "proximate" level and the "ultimate" level, in evolutionary explanatory taxonomy, Mayr 1993), the problem dissolves. Some would argue that felt experiences are causally "epiphenomenal" and hence play no causal role in the process of adaptation; but even if this were so, it would not imply that adaptive behavior cannot provide evidence for felt states, so long as we have reason to believe that felt states accompany certain bodily states.

The adaptive value of sentient states may cut against another source of skepticism about felt states in non-human animals: the existence of robots that replicate nociceptive behavior (Cartmill). How can we infer that biological mice feel pain while (current) robot mice do not, even though both exhibit similar withdrawal behaviors in response to detected damage? The robot case makes much of the biological machinery look unnecessarily elaborate and superfluous, even though natural selection will tend to economize. As Elwood points out, robots are human contrivances that are very different from organisms equipped by natural selection with complex motivational systems and their attendant behaviors. (Balcombe makes a similar point.) That valenced feeling (including pain perception) has evolved is perhaps our best explanation of this "extra" machinery.

Evidence for sentient states such as pain does not come from behavior alone: that ants exhibit responses to analgesics and antidepressants similar to those of humans, as Cammaerts suggests, further bolsters the case that they are sentient and weakens the argument from robotics. Withdrawal from and avoidance of noxious stimuli (Zentall) combined with responsiveness to anesthetics and analgesics provide evidence of pain perception across all animal groups, especially when coupled with neurobiological features such as nociception and the presence of endogenous opioids. The contrived robotic mimicry of pain-like behavior and other affective states can be seen as proof of concept for insentient damage-avoidance-but it does not provide evidence of how blind variation and natural selection operates in the 'real' biological world.

3.5 Beyond Pain: The Conditions for Flourishing. We stressed in our target article that pain is not the only sentient state that matters morally. Several commentators (Woodruff; Vonk; Baracci \& Baciadonna) agree that a wide range of affective states beyond the sensation of pain and suffering need to be considered, including those that contribute to animal "flourishing" (Nussbaum 2006); at the same time, they highlight the difficulty of tracking those states empirically.

Identifying the functional markers of flourishing across great phylogenetic distances may be even more challenging than the problem of pain perception. As Woodruff remarks, understanding how these alien species can flourish may be more daunting than determining whether they are sentient. In contrast to pain perception, it is possible that no functional markers for flourishing exist across phyla; it may vary tremendously from a mouse to a mantid, from individual to individual within a species (Vonk) and even across transformative life stages and historical trajectories of an individual invertebrate animal.

Although the conditions for flourishing are context-dependent phylogenetically, developmentally, and ecologically, it may be possible to devise a general-purpose strategy 
for identifying them species-specifically. Wemeslfelder (2007) ${ }^{1}$ proposes a framework for studying 'quality of life' across species by drawing on the expertise of animal researchers and caretakers. These are best positioned to interpret specific body language in light of their familiarity with the animal's behavior, ecology, and individual history. The idea is that ethology should follow ethnology by embedding the observer in the Umwelt of the observed, illuminating aspects of animal experience that a "dispassionate" observer might have overlooked (Andrews 2009; 2020).

One might wonder whether the quality-of-life approach can be usefully extended to invertebrates whose motivational states are especially difficult to read in their unfamiliar behaviors. Those who actually work with invertebrates do not find this difficulty insurmountable. Balcombe notes that the more we learn about arthropods like insects, the more we come to recognize their welfare. Researchers themselves are arguably the ones who are best positioned to map out the conditions under which these alien lives are well lived.

\section{Balancing Scientific Uncertainty and Moral Risk}

4.1 Due Precaution Without a Precautionary Principle. We share the ethical concerns that motivate precautionary approaches to animal sentience, and we recognize the value of avoiding the need to establish sentience one species at a time. However, we do not believe that a precautionary principle is the best way of managing moral risk in this context In our target article we suggest that Birch's (2017) proposed precautionary principle (ASPP), which he tailors to the problem of animal sentience, requires more than the pragmatic argument that a case-by-case analysis of sentience in lower-level taxa (species, genera, etc.) is not feasible. Choosing the Linnaean order as the relevant grain of phylogenetic resolution, as the ASPP does, we argued, requires a theoretical defense. This choice only makes sense if we assume (1) that the last common ancestor of the order was sentient and (2) that the conservation of sentience is more likely than its loss in a given order. There is then the further problem that establishing the polarity of a trait in a higher-level clade requires a caseby-case analysis of the presence or absence of that trait among the lower-level taxa that comprise the clade.

Birch insists that he does not need to defend any of these substantive theoretical commitments. All he needs to do is show that "if evidence of sentience is found in one species then the probability of sentience in other species of the same order is thereby raised-and that this upward shift in the probability of sentience pragmatically justifies taking precautions." However, this opens the ASPP up to conceptual problems that may result in over-including or under-including sentient organisms, as we shall now explain. The mere fact that some piece of evidence increases the probability of sentience is not sufficient to justify precautionary measures. If it were, then there would be no reason to limit the ASPP to Linnaean orders. The fact that jellyfish have nervous systems increases the probability that they are sentient, even if that probability is small; the fact that green plants have complex molecular signaling and information transfer mechanisms increases the probability they are sentient, even if that probability is small; and to take the logic to the extreme, the bare fact of being alive increases the probability that a biological system is sentient, even if that probability is extremely low. If a mere uptick in probability were sufficient to trigger

\footnotetext{
${ }^{1}$ We are grateful to Michael Woodruff for directing us to this work.
} 
precautionary measures, then why not apply the ASPP to all metazoans (as Veit \& Huebner recommend), to all multicellular eukaryotes, or better yet, to all living things (as Dietrich $\boldsymbol{\&}$ Fox Hall suggest)? There must be a baseline threshold of plausibility. Of course, if we knew what structures were sufficient for sentience we could rule some or all of these cases out. But if we could do that, then we would have no need for a course-grained heuristic like the ASPP.

To put the problem another way: many different types of evidence bear on the probability of sentience in a given clade, so why limit the precautionary trigger to phylogenetic information? The ASPP creates a reasonable "taxonomic zone of precaution" that recognizes our current epistemic limitations. But because of its reliance on taxonomy, the ASPP ends up being too permissive in some cases and too restrictive in others. It is too permissive where order-level clades are especially diverse in their anatomy, behavior, development, and lifeways. Balcombe notes that this is true of many higher arthropod taxa. In other cases, it is overly restrictive: for example, it would not allow credible findings of sentience in octopods to generalize to all coleoids (such as decapods, which include living squid and cuttlefish and extinct belemnoids), let alone to other cephalopod orders like the extinct ammonites, which are comparable in their neural anatomy and behavioral ecology.

In short, the ASPP shares a flaw with other precautionary principles: it discourages evidence gathering to the detriment of rational risk management. It asks us to ignore troves of existing data and theory in the service of a workable and compassionate but insufficiently defended taxonomic heuristic. What we need is an approach that is contextual and evidencesensitive, not course-grained and indefeasible. We agree wholeheartedly that sentience should not have to be proven on a species-by-species basis before animals can be afforded reasonable protections. But substituting one hard-and-fast principle for another is not, in our view, the best way forward. Instead, we recommend a more context-sensitive approach that takes into account not only experimentally elicited behavior, but also trait polarity, neuroanatomy, and behavioral ecology in making judgments about the sentience of clades (see Chapouthier). We acknowledge that our alternative is more burdensome from the standpoint of ethical policy. But given what is at stake, we believe the tradeoff between speed and accuracy weighs in favor of getting the attribution of sentience right.

4.2 The Moral Costs of Overattribution. Morality entails risk. The under-attribution of sentience comes with significant moral costs to the sentient beings who are denied moral protections. But the moral costs associated with over-attribution are not trivial either: there are thus strong reasons to get the attribution right. This side of the risk equation is overlooked by several commentators.

Lockwood summarizes our argument as a modified version of Pascal's wager, but he erroneously sets the pay-off matrix such that acting ethically toward non-sentient beings entails no moral loss. Soryl argues that given the vast numbers of potentially sentient invertebrates like arthropods, even the mere possibility of invertebrate sentience should speak in favor of including them in the moral calculus, given the costs of wrongly denying them moral protections. But since there is a very real possibility (however remote) that brainless animals with ganglionic or distributed nervous systems are also sentient, why not include them in the moral calculus as well? This seems to be the logic driving Veit $\boldsymbol{\&}$ Huebner, who think that erring on the side of caution requires an even more profound shift in inclusiveness than we entertain, namely, extending moral protections to all non-human 
animals, including those without brains. But if there are no major costs to over-attribution, why stop there? Why not include animals without nervous systems, like sponges? Why not green plants and unicellular eukaryotes? We need to rein this in lest we conclude that human herbivores are no less morally culpable for eating plants than are meat-eaters for eating meat -- which brings us (and others, such as Marino and Mallatt \& Feinberg) to centralized nervous systems as the appropriate risk calibration point. Dietrich \& Fox Hall may be right that it is impossible to do no harm to other sentient creatures given how the biological world is structured; but doing our moral best requires getting the attribution of sentience to be as accurate as possible, given our current state of scientific and moral knowledge.

Lockwood does draw attention to an important and overlooked benefit that could flow from acting as if some group of beings had moral standing when in fact they do not: namely, that doing so may have benefits for human moral character, such as the development of compassion, kindness, epistemic humility, and so forth. These virtues can either be valuable in and of themselves, or they can have instrumental value if they improve our treatment of other beings who do have moral standing. Lockwood still requires that our beliefs about sentience be "reasonable" - presumably to avoid practicing kindness toward things that clearly do not warrant it, such as stuffed animals or pet rocks, things that lack the capacity to suffer or flourish. But this reasonableness criterion draws on the same subjectcentered view of morality that we advocate and that he claims is inferior to the virtue-ethical approach. Both subject-centered and virtue-ethical approaches are constrained by reasonable beliefs about who matters morally and why, since both are trying to make reasonable assessments of risk.

4.3 Aggregation Problems. Attention is drawn by Soryl to invertebrate suffering in the wild. He is right that the idea of a benevolent balance in nature is a myth that glosses over the incalculable suffering that occurs in nature irrespective of human agency (Powell 2011). Nature is amoral and natural selection does not have animal welfare at heart. Soryl appears to proceed from the fact of natural suffering to the idea that an analysis in term of aggregate utility dictates intervening in nature on behalf of invertebrate welfare, perhaps even requiring that we give priority to such interventions over efforts to prevent the suffering of vertebrates such as pigs, cows, and chickens at the hands of humans in agricultural settings. There seem to be two fundamental problems with this argument:

The first problem is with comparing welfare between persons or between organisms. It has been accepted for nearly a century that the early Utilitarian attempt to quantify welfare as a single measurable unit aggregated within and between lives did not, and probably cannot, succeed. There is no known objective way to make quantitative interpersonal welfare comparisons; welfares are 'non-additive' in that they cannot be summed to arrive at a number for total welfare. If welfare cannot be summed, then it cannot be maximized. And if welfare cannot be measured or maximized across humans, then there is virtually no chance this could be done across creatures as different as arthropods and mollusks, whose functional markers of subjective wellbeing are, as noted earlier, much harder to assess.

The second problem with intervening in nature to promote utility is that there are sound reasons to focus our ethical efforts on anthropogenic harms rather than on natural states of affairs, quite apart from the unintended harmful effects of human intervention. It is widely accepted (Pogge 2001) that moral agents have stronger duties not to harm than to help. Outright murder is near universally regarded as worse, morally speaking, than failing 
to aid people in need, especially those to whom we bear no proximal relationship of care (unlike, say, our children). Although this asymmetry is often grounded in Kantian moral philosophy, it can also be defended on Utilitarian grounds.

A further dimension of the aggregation problem, noted by Monsó \& Osuna-Mascaró, concerns how to weigh the interests of a great many individuals of lower moral status (such as sentient nonpersons) against a smaller number of individuals with higher moral status (such as sentient persons). This problem is not unique to Utilitarianism; it is shared by all moral theories. Is it preferable to give priority to the welfare of a handful of mammalian individuals over the suffering of a large number of insect individuals? This question arises poignantly in the agricultural context: for example, soybean farming is often thought to be ethically preferable to the farming of vertebrate animals; yet in the process untold numbers of arthropods are made to suffer. These conflicts may be genuine "moral dilemmas" in the technical sense that they have no correct solution, at least given our current understanding of morality and what is practically possible in the world.

4.4 The Jain Monk Objection. The sheer number of arthropods with potential welfares to care about leads to another moral concern: that taking invertebrate interests into account leads to morally unpalatable sacrifice. Forber $\&$ Jones remark that the result of a simple stroll down a country road at night is no less than arthropod slaughter. (This is far less of a problem, we would note, for coleoid mollusks, with whom we do not "share" our daily lives.) Would according moral standing to arthropods imply that we all become Jain Monks, as Carruthers (2007) maintains?

We think not. As Browning \& Veit point out, mundane human activities such as cardriving harm not only arthropods, but also other vertebrates-including humans-and yet we accept these moral costs as long as reasonable precautions are taken. Even if we need not sweep the floor before us for fear of stepping on foraging ants and beetles, this does not give us the moral license to deliberately or wantonly step on these animals as we walk, or to pay no heed to their welfare in the laboratory (DeGrazia). There is also reason to hold researchers to a higher moral standard than members of the lay population, for many reasons: Researchers are more familiar with the minds and behaviors of the animals they study; they seek out and control the conditions under which they interact with these animals; and they serve as important moral models for the public at large, as illustrated by their advocacy on behalf of conservation, climate change initiatives, and the ethical treatment of animals.

\section{Changing Hearts and Minds}

5.1 Biases and Intuitions. Scientific research will not make a material difference to the lives of animals if it is not acted upon by policy-makers. What is the most effective way to promote policies grounded in science and ethics rather than political expediency? Several commentators suggest that in order to reshape the landscape of invertebrate ethics, we must first change the minds (and hearts!) of the lay population through science outreach and education. Balcombe suggests that the most effective way to reframe lay moral intuitions is to train people to be watchers of animals-in effect, transforming them into expert observers capable of 'seeing' the minds and welfares of other creatures. Educating people about the 
impressive capabilities of arthropods and their ecological roles may go some way toward reshaping public opinion of these animals. Abstract scientific knowledge is only part of what makes for careful observation, however; also crucial are motivation, compassion, humility, awe, and imagination.

To this end, Marzluff recommends that scientists collaborate with story-tellers such as poets and artists, and that they take advantage of a variety of platforms including not only public lectures and opinion pieces, but documentaries, social media, and early childhood nature education. We could not agree more. There are important questions about the proper roles of emotion and intuition in science, in morality, and in policy-making. In our target article we argued that cognitive biases may cause us to under-attribute mental states and moral value to animals who fail to garner our sympathy, such as beings we find disgusting, unfamiliar, or threatening. But as the foregoing discussion suggests, emotion and intuition can also help us locate sentient minds in nature and acknowledge their moral force.

Funding: The authors are grateful to Templeton World Charity Foundation grant \# 0469 for support of this research.

Acknowledgements: We are especially appreciative of the Herculean efforts of the editor, Stevan Harnad, to avoid verbal confusion, communicative barriers, and rhetorical red-herrings that could impede progress on these scientifically challenging and ethically important issues.

\section{References}

Abramson, C. I., \& Chicas-Mosier, A. M. (2016). Learning in plants: lessons from Mimosa pudica. Frontiers in Psychology, 7, 417.

Adler, J. (1966). Chemotaxis in bacteria. Science, 153(3737), 708-716.Agar, N. (2013). Truly human enhancement: a philosophical defense of limits. MIT Press.

Amundson, R. \& Lauder, G.V. (1994). Function without purpose. Biology and Philosophy 9(4): 443-469.

Andrews, K. (2020). How to study animal minds. Cambridge University Press.

Andrews, K. (2009). Politics or metaphysics? On attributing psychological properties to animals. Biology \& Philosophy 24(1): 51-63.

Balcombe, J. (2020) Intuition and the invertebrate dogma. Animal Sentience 29(9).

Baracchi, D. \& Baciadonna, L. (2020) Insect sentience and the rise of a new inclusive ethics. Animal Sentience 29(18).

Basl, J. (2019). The death of the ethic of life. Oxford University Press.

Bayne, T., Hohwy, J., \& Owen, A.M. (2016). Are there levels of consciousness? Trends in Cognitive Sciences 20(6): 405-413.

Birch, J. (2020) Zones of precaution. Animal Sentience 29(28).

Birch, J. (2017). Animal sentience and the precautionary principle. Animal Sentience 16(1).

Broom, D.M. (2020) Brain complexity, sentience and welfare. Animal Sentience 29(27). 
Brown, C. (2016). Comparative evolutionary approach to pain perception in fishes. Animal Sentience 3(5).

Brown, C. (2020). Convergent evolution of sentience? Animal Sentience 29(25).

Browning, H. \& Veit, W. (2020) Improving invertebrate welfare. Animal Sentience 29(4).

Buchanan, A. (2011). Better than human: the promise and perils of enhancing ourselves. Oxford University Press.

Cammaerts, M.C. (2020) Invertebrates should be given ethical consideration. Animal Sentience 29(6.)

Carruthers, P. (2007). Invertebrate minds: a challenge for ethical theory. The Journal of Ethics 11(3): 275-297.

Cartmill, M. (2020) Do beetles have experiences? How can we tell? Animal Sentience 29(31).

Cartmill, M. (2000). Animal consciousness: some philosophical, methodological, and evolutionary problems. American Zoologist, 40(6): 835-846.

Chapman, Colin A. and Huffman, Michael A. (2018) Why do we want to think humans are different? Animal Sentience 23(1)

Chapouthier, G. (2020) Invertebrate cognition, sentience and biology. Animal Sentience 29(26).

Crisp, R. (2020) Invertebrate ethics. Practical Ethics. Oxford Blog.

DeGrazia, D. (2020). On the possibility of invertebrate sentience. Animal Sentience 29(15).

Delon, N. et al. (2020) Consider the agent in the arthropod. Animal Sentience 29(32).

De Waal, F B. (1999). Anthropomorphism and anthropodenial: consistency in our thinking about humans and other animals. Philosophical Topics 27(1): 255-280.

Dietrich, E. and Fox Hall, T. (2020). Moral treatment for all. Animal Sentience 29(23).

Dinets, V. (2016). No cortex, no cry. Animal Sentience 3(7)

Elwood, R.W. (2020) Do arthropods respond to noxious stimuli purely by reflex? Animal Sentience 29(10).

Figdor, C. (2020) Relationship between cognition and moral status needs overhaul. Animal Sentience 29(3).

Fitzpatrick, sentience.(2020) Avoiding anthropocentrism in evolutionarily inclusive ethics. Animal Sentience 29(29).

Forber, P. \& Jones, R.C. (2020) Spineless and sentient: a challenge for moral comparison. Animal Sentience 29(30).

Friedman, D.A. \& Søvik, E. (2019). The ant colony as a test for scientific theories of consciousness. Synthese 1-24.

Gibbons, M. \& Sarlak, sentience.(2020). Inhibition of pain or response to injury in invertebrates and vertebrates. Animal Sentience 29(34).

Howard, sentience.R. \& Symonds, M.R.E. (2020) Ethical considerations for invertebrates. Animal Sentience 29(21). 
Jarvis, E.D., et al. (2005). Avian brains and a new understanding of vertebrate brain evolution. Nature Reviews Neuroscience 6(2): 151-159.

Kennedy, J.S. (1992). The new anthropomorphism. Cambridge University Press.

Key, B. (2016). Falsifying the null hypothesis that "fish do not feel pain. Animal Sentience: An Interdisciplinary Journal on Animal Feeling 3(39).

Key, B. and Brown, D. (2020) Minds, morality and midgies. Animal Sentience 29(24).

Lecky, W.E.H. (1921) History of European Morals from Augustus to Charlemagne, vol. 1, 3rd ed. New York: Appleton.

Lee, A.Y. (2020). Does sentience come in degrees? Animal Sentience 29(20).

Levy, N. (2020) It might not matter very much whether insects are conscious. Animal Sentience 29(2)

Lockwood, J.A. (2020) Virtue ethics and the likelihood of invertebrate suffering. Animal Sentience 29(12).

Mallatt, J. and Feinberg, T.E. (2020) Sentience in evolutionary context. Animal Sentience 29(14)

Marino, L. (2020). Sentience in all organisms with centralized nervous systems? Animal Sentience 29(19).

Marzluff, J.M. (2020) Bridging the empathy gap for invertebrates. Animal Sentience 29(22).

Mayr, E. (1993). Proximate and ultimate causations. Biology and Philosophy 8(1): 93-94

McMahan, J. (2002). The ethics of killing: Problems at the margins of life. Oxford University Press, USA.

McShea, D.W. (2013). "Machine wanting." Studies in History and Philosophy of Science Part C: Studies in History and Philosophy of Biological and Biomedical Sciences 44(4): 679687.

Mikhalevich, I. (2017). Avoiding old mistakes in new experimental contexts. The Routledge Handbook of Philosophy of Animal Minds 427.

Mikhalevich, I. (2015). Experiment and animal minds: why the choice of the null hypothesis matters. Philosophy of Science 82(5): 1059-1069.

Mikhalevich, I. (2014). A critique of the principle of cognitive simplicity in comparative cognition. Biology \& Philosophy 29(5): 731-745 (published under Meketa).

Mikhalevich, Irina and Powell, Russell (2020) Minds without spines: Evolutionarily inclusive animal ethics. Animal Sentience 29(1

Monsó, sentience.and Osuna-Mascaró, A.J. (2020) Problems with basing insect ethics on individuals' welfare. Animal Sentience 29(8).

Morgan CL (1893). "Other minds than ours . An Introduction to Comparative Psychology. London: Scott.

Neander, K. (2002). 15. Types of Traits: The Importance of Functional Homologues. Functions: New essays in the philosophy of psychology and biology, 390.

Neander, K. \& Rosenberg, A. (2012). Solving the circularity problem for functions: A response to Nanay. The Journal of philosophy 109(10): 613-622. 
Nussbaum, M. C. (2006). The moral status of animals. Chronicle of Higher Education 52(22), B6-8.

Pepperberg, I.M., \& Pepperberg, I. M. (2009). The Alex studies: cognitive and communicative abilities of grey parrots. Harvard University Press.

Pogge, T. (2001). Priorities of global justice. Metaphilosophy, 32(1-2), 6-24.

Powell, R. (2020). Contingency and convergence: Toward a cosmic biology of body and mind. MIT Press.

Powell, R. (2010). What's the Harm?: An Evolutionary Theoretical Critique of the Precautionary Principle. Kennedy Institute of Ethics Journal 20(2): 181-206.

Powell, R., Mikhalevich, I. \& Buchanan, A. (in press). How the moral community evolves. In Rethinking Moral Status, Clarke, sentience.and Savulescu, J. (eds.), Oxford University Press.

Reid, C. R., Latty, T., Dussutour, A., \& Beekman, M. (2012). Slime mold uses an externalized spatial "memory" to navigate in complex environments. Proceedings of the National Academy of Sciences, 109(43), 17490-17494.

Rose, J. D. (2002). The neurobehavioral nature of fishes and the question of awareness and pain. Reviews in Fisheries Science 10(1): 1-38.

Rosenberg, A., \& Neander, K. (2009). Are homologies (selected effect or causal role) function free? Philosophy of Science 76(3): 307-334.

Schwitzgebel, E. (2015). If materialism is true, the United States is probably conscious. Philosophical Studies 172(7): 1697-1721.

Sober, E. (2005). Comparative psychology meets evolutionary biology. Thinking with animals: New perspectives on anthropomorphism, 85.

Soryl, A. (2020) Invertebrate welfare in the wild. Animal Sentience 29(7)

Tononi, G., \& Koch, C. (2015). Consciousness: here, there and everywhere?. Philosophical Transactions of the Royal Society B: Biological Sciences, 370(1668), 20140167.

Vallortigara, G. (2020) Lessons from miniature brains: Cognition cheap, memory expensive (sentience linked to active movement?) Animal Sentience 29(17).

Veit, W. \& Huebner, B. (2020) Drawing the boundaries of animal sentience. Animal Sentience 29(13).

Vonk, J. (2020) No room for speciesism in welfare considerations. Animal Sentience 29(5).

Wemelsfelder, F. (2007). How animals communicate quality of life: the qualitative assessment of behaviour. Animal Welfare 16 (Supp. 1): 25-31.

Woodruff, M.L. (2020) Whether invertebrates are sentient matters to bioethics and science policy. Animal Sentience 29(16).

Zentall, T.R. (2020). Cognition, movement and morality. Animal Sentience 29(11). 